\title{
Different fracture risk profile in patients treated with anti-osteoporotic drugs in real-life
}

\author{
G. Adami, A. Giollo, M. Rossini, G. Orsolini, C. Benini, O. Viapiana, \\ D. Gatti, A. Fassio \\ Rheumatology Unit, University of Verona, Policlinico Borgo Roma, Verona, Italy
}

\begin{abstract}
SUMMARY
In this retrospective study, we intended to investigate the baseline fracture risk profile in patients who started treatment with different anti-osteoporotic medications.

We analyzed retrospectively the fracture risk calculated with DeFRA, a validated FRAX derived tool, in women who started an anti-osteoporotic treatment from 2010 to 2017.

We analyzed baseline data of 12,024 post-menopausal women aged over 50 years. Teriparatide initiators had a baseline 10-year risk of major osteoporotic fracture of $82.1 \%$ with a Standard Deviation (SD) of 66.5\%. Denosumab initiators and zoledronic acid initiators had a greater 10 -year baseline risk of fracture $(54.3 \%, \mathrm{SD} 46.5 \%$ and $47.0 \%$, SD 42.0 respectively) than patients initiated on alendronate (24.9\%, SD 34.6\%) and patients initiated on risedronate $(23.9 \%$, SD $24.1 \%)$.

Using DeFRA, a FRAX ${ }^{\mathrm{TM}}$ derived tool, we showed significantly different fracture risk profiles in women who were started on various therapeutic agents for the treatment of osteoporosis in routine clinical practice.
\end{abstract}

Key words: Risk of fracture; anti-osteoporotic medications.

Reumatismo, 2020; 72 (2): 71-74

\section{INTRODUCTION}

$\mathrm{M}$ edications that suppress bone resorption, including bisphosphonates and denosumab, and anabolic agents, such as teriparatide, are commonly prescribed for the treatment of osteoporosis. Randomized clinical trials demonstrated that teriparatide and denosumab are more effective than bisphosphonates in increasing BMD (1-3). Nevertheless, data from real-life practice showed a substantial equivalence in terms of prevention of fragility fractures between denosumab and alendronate (4). It is unclear whether denosumab, in routine clinical practice, is prescribed to patients with a greater risk of fracture compared to alendronate and other bisphosphonates. The Fracture Risk Assessment Tool (FRAX ${ }^{\mathrm{TM}}$ ) developed by the WHO Collaborating Centre for Metabolic Bone Diseases represents a widely used tool for the calculation of fracture risk (5). DeFRA is a new, webbased, open source, FRAX ${ }^{\mathrm{TM}}$ derived tool used to assess the 10-year risk of fracture (6). DeFRA tool comprises several variables: age, weight, height, number and site of prior fragility fracture, family history of hip and clinical vertebral fracture, smoking status (as semiquantitative variable), alcohol intake (as semiquantitative variable), glucocorticoid intake (as semiquantitative variable), diagnosis of rheumatoid arthritis, systemic lupus erythematosus, psoriatic arthritis, scleroderma and other connective tissue diseases and both hip and lumbar spine BMD values $(6,7)$. The aim of this study is to determine the baseline fractures risk profile in patients who were started on different anti-osteoporotic drugs.

\section{MATERIALS AND METHODS}

We analyzed retrospectively the 10-year risk of major osteoporotic fractures predicted by the DeFRA tool in post-menopausal women aged over 50 years who started an anti-osteoporotic treatment from
Corresponding author: Giovanni Adami

Rheumatology Unit, University of Verona Policlinico Borgo Roma, Verona, Italy E-mail: adami.g@yahoo.com 
2010 to 2017. DeFRA database comprises more than 120,000 patients at high risk of fracture. In this study we enrolled women who were seen by 100 Italian bone specialists (including mainly endocrinologists and rheumatologists) and treated with teriparatide and denosumab as well as other antiosteoporotic therapies. The bone specialists were based in various regions across Italy (45\% northern Italy, $25 \%$ central Italy, $30 \%$ southern Italy). Subjects with incomplete collection of data were excluded from the analysis. All the women were identified in the database with an anonymous code, which was linked to the medical records, in full compliance with the Italian code for the protection of personal data. No identifiers related to patients were provided to the researchers. The results of analyses were shown as aggregated summaries, which were impossible to assign, either directly or indirectly, to the individual patients. This study was approved by the local ethical committee. We used descriptive statistics to characterize the subjects included in this study. Means (SD) were calculated for normally distributed continuous variables and proportions were used to describe categorical variables. The differences between groups were tested with independent-samples $t$-test and were considered significant when the $p$ value was below 0.05. All statistical analyses were performed using SPSS Version 20 (SPSS, Inc., Chicago, IL, USA).

\section{RESULTS}

We retrieved data for 12,024 women who were initiated on an anti-osteoporotic treat-

Table I - Descriptive characteristics of 12,024 women stratified by prescribed treatment at the time of the visit.

\begin{tabular}{|c|c|c|c|c|c|c|}
\hline \multicolumn{2}{|l|}{ 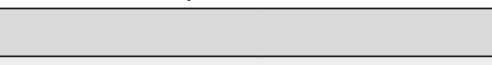 } & Alendronate & Risedronate & Zoledronic acid & Denosumab & Teriparatide \\
\hline \multicolumn{2}{|l|}{$\mathrm{N}$, total $=12,024$} & 7,288 & 1,592 & 338 & 2,247 & 559 \\
\hline \multicolumn{2}{|l|}{ Age, years (mean $\pm S D$ ) } & $69 \pm 9$ & $68 \pm 9$ & $73 \pm 9$ & $72 \pm 9$ & $73 \pm 9$ \\
\hline \multicolumn{2}{|c|}{ Total Hip T-score $($ mean \pm SD) } & $-2.4 \pm 0.8$ & $-2.4 \pm 0.8$ & $-2.3 \pm 0.9$ & $-2.6 \pm 0.9$ & $-2.6 \pm 1.0$ \\
\hline \multirow{2}{*}{ Smoking } & No & $84.5 \%$ & $86.6 \%$ & $87.9 \%$ & $85.8 \%$ & $86.0 \%$ \\
\hline & Yes & $15.5 \%$ & $13.4 \%$ & $12.1 \%$ & $14.2 \%$ & $14.0 \%$ \\
\hline \multirow{2}{*}{$\begin{array}{l}\text { Alcohol consumption } \\
\text { (>10 U per day) }\end{array}$} & No & $99.1 \%$ & $99.6 \%$ & $95.6 \%$ & $99.2 \%$ & $98.7 \%$ \\
\hline & Yes & $0.9 \%$ & $0.4 \%$ & $4.4 \%$ & $0.8 \%$ & $1.3 \%$ \\
\hline \multirow{3}{*}{$\begin{array}{l}\text { Prednisone } \\
\text { equivalent intake }\end{array}$} & $>2.5<5 \mathrm{mg}$ & $8.2 \%$ & $7.7 \%$ & $11.2 \%$ & $5.8 \%$ & $12.0 \%$ \\
\hline & $\geq 5 \mathrm{mg}$ & $3.6 \%$ & $2.8 \%$ & $9.5 \%$ & $3.4 \%$ & $9.1 \%$ \\
\hline & No & $88.2 \%$ & $89.5 \%$ & $79.3 \%$ & $90.7 \%$ & $78.9 \%$ \\
\hline \multirow{2}{*}{ Falls in the last year } & No & $87.5 \%$ & $90.1 \%$ & $84.0 \%$ & $88.4 \%$ & $83.5 \%$ \\
\hline & Yes & $12.5 \%$ & $9.9 \%$ & $16.0 \%$ & $11.6 \%$ & $16.5 \%$ \\
\hline \multirow{5}{*}{ Vitamin D intake } & $<250$ U die & $41.6 \%$ & $30.8 \%$ & $27.5 \%$ & $35.1 \%$ & $31.3 \%$ \\
\hline & 250-400 U die & $7.5 \%$ & $6.4 \%$ & $7.7 \%$ & $6.9 \%$ & $6.6 \%$ \\
\hline & $400-800$ U die & $22.8 \%$ & $28.2 \%$ & $34.9 \%$ & $31.8 \%$ & $32.4 \%$ \\
\hline & $800-1200$ U die & $20.8 \%$ & $23.9 \%$ & $19.8 \%$ & $17.6 \%$ & $19.3 \%$ \\
\hline & $>1200$ U die & $7.3 \%$ & $10.7 \%$ & $10.1 \%$ & $8.6 \%$ & $10.4 \%$ \\
\hline \multirow{2}{*}{$\begin{array}{l}\text { Family history of } \\
\text { femoral fracture }\end{array}$} & No & $75.0 \%$ & $71.6 \%$ & $79.3 \%$ & $70.4 \%$ & $69.9 \%$ \\
\hline & Yes & $25.0 \%$ & $28.4 \%$ & $20.7 \%$ & $29.6 \%$ & $30.1 \%$ \\
\hline \multirow{2}{*}{$\begin{array}{l}\text { Prior clinical major } \\
\text { fracture }\end{array}$} & No & $65.2 \%$ & $64.1 \%$ & $36.1 \%$ & $22.1 \%$ & $5.5 \%$ \\
\hline & Yes & $34.8 \%$ & $35.9 \%$ & $63.9 \%$ & $77.9 \%$ & $94.5 \%$ \\
\hline \multicolumn{2}{|c|}{$\begin{array}{l}\text { \% estimated } 10 \text {-year major fracture risk } \\
\text { (mean } \pm \text { SD) }\end{array}$} & $24.9 \pm 34.6$ & $23.9 \pm 24.1$ & $47.0 \pm 42.0$ & $54.3 \pm 46.5$ & $82.1 \pm 66.5$ \\
\hline \multicolumn{2}{|c|}{$\begin{array}{l}\% \text { estimated } 10 \text {-year femoral fracture risk } \\
\text { (mean } \pm \text { SD) }\end{array}$} & $11.5 \pm 36.9$ & $10.5 \pm 14.2$ & $21.9 \pm 26.8$ & $29.0 \pm 65.4$ & $44.8 \pm 60.6$ \\
\hline
\end{tabular}




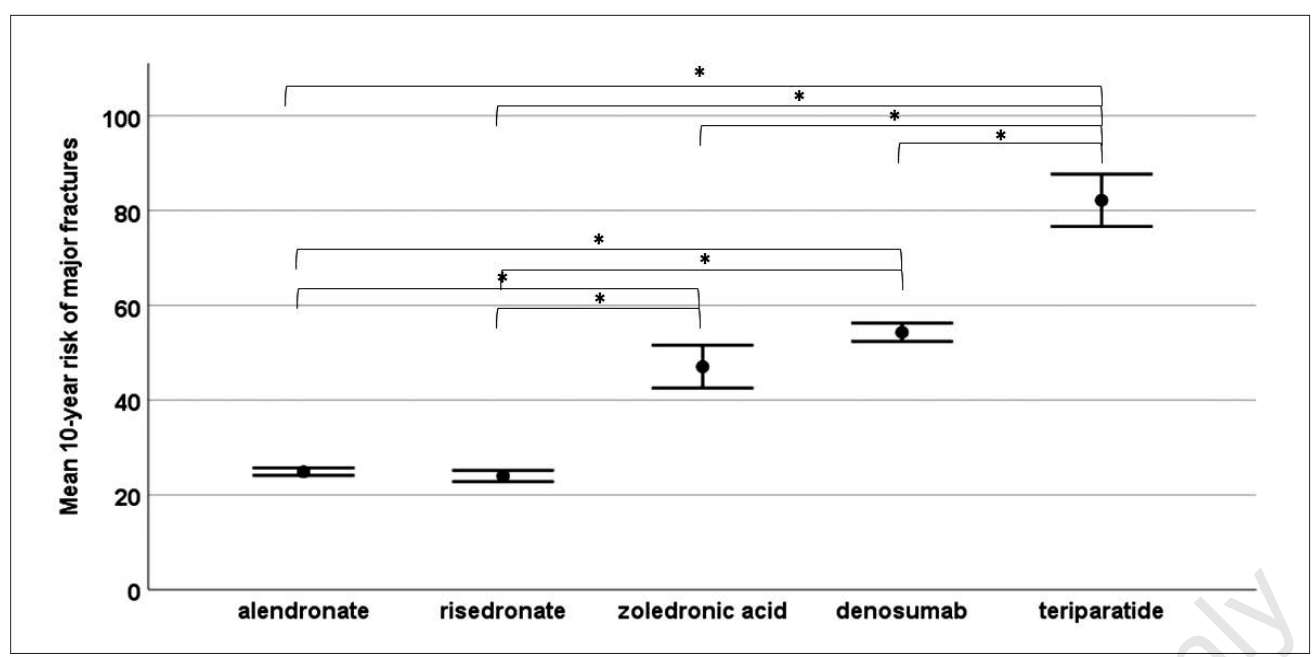

Figure 1 - Mean 10-year fracture risk estimated with DeFRA tool at the time of treatment initiation, ${ }^{*} p<0.01$.

ment at the time of the visit. Table I shows the baseline characteristics of women stratified by prescribed medication. Figure 1 shows the mean $[95 \%$ confidence interval (CI)] 10-year risk of major osteoporotic fractures estimated with the DeFRA tool at the time of treatment initiation. Teriparatide users had the highest 10-year risk of fracture (82.1\% Standard Deviation (SD) $66.5 \%$ ). We found that in 2,247 patients started on denosumab the 10-year baseline risk of fracture $(54.3 \%, \mathrm{SD} 46.5 \%)$ was significantly greater than in 7,288 patients initiated on alendronate $(24.9 \%$, SD $34.6 \%)$ and in patients initiated on risedronate $(23.9 \%$, SD $24.1 \%)$. Patients starting zoledronic acid had a similar 10-year risk of fracture compared to those starting denosumab (47.0\%, SD 42.0). Similar results were found for the 10-year risk of hip fracture (data not shown).

\section{DISCUSSION}

In this analysis, we described the 10-year fracture risk profile of patients who were initiated on different anti-osteoporotic medications. Unsurprisingly, a new prescription of teriparatide was associated with the highest risk of fracture, which was approximately four-fold greater than oral bisphosphonates. This result is in line with several national and international guidelines, including the Italian guideline, which recommends to start bone anabolic agents in patients with severe osteoporosis who experienced at least one or more fragility fracture $(7,8)$. Indeed, the majority of teriparatide users had at least one prior fragility fracture. However, around $5.5 \%$ of teriparatide users did not experience any fragility fracture, therefore its prescription was not reimbursed by the Italian regulatory agency. Patients with a new prescription of denosumab had a two-fold higher 10-year risk of fracture compared to patients initiated on oral bisphosphonates. In particular, a similar incidence of fragility fracture with the use of alendronate compared to denosumab has been recently reported by Pedersen et al. (4). This finding is somewhat surprising, given that denosumab has been associated with a sustained and linear increase in BMD (9). Moreover, denosumab suppresses bone turnover and increases BMD more than alendronate and should be associated with a lower incidence of fractures $(10,11)$. Regrettably, Pedersen and colleagues could not include important variables in their analysis (i.e., baseline BMD values). Our findings suggest that, in routine clinical practice, patients started on teriparatide, denosumab and zoledronic acid have a greater risk of fracture compared to individuals receiving oral bisphosphonates. Due to confounding indications, a type of channeling bias might have biased the study by Pedersen and colleagues. Indeed, as 
demonstrated by our analysis, denosumab is commonly prescribed to patients with severe osteoporosis, who are at a greater risk of fracture.

Our study has both strengths and limitations. We analyzed the fracture risk with a validated tool in many individuals who were evaluated by bone specialists. However, our findings cannot be generalized and applied to other countries in which physicians might follow different treatment algorithms. Furthermore, denosumab started to be marketed in 2011 in Italy, hence we had a 1-year gap for denosumab prescription that could have possibly altered the analysis.

\section{CONCLUSIONS}

In summary, teriparatide, denosumab and zoledronic acid are prescribed to patients at greater risk of fracture. For this reason, real-life treatment effectiveness of teriparatide, denosumab and zoledronic acid might not be comparable to other anti-osteoporotic medications. Non-randomized, comparative studies on the effectiveness of various anti-osteoporotic medication should always consider the baseline fracture risk profile. An accurate, integrated, assessment of multiple risk factors is recommended.

\section{Conflict of interest}

The authors declare that they have no conflict of interest.

\section{Ethical Statement}

All the subjects under analysis were identified in the database by an anonymous code, which was linked to the medical records, in full compliance with the Italian code for the protection of personal data (Legislative Decree 196/03, http://www.camera.it/parlam/leggi/deleghe/03196dl.htm). All procedures performed in studies involving human participants were conducted in accordance with the ethical standards of the institutional and/or national research committee and with the 1964 Helsinki Declaration and its later amendments or comparable ethical standards. Informed consent was not required for this study and no identifiers related to patients were provided to the researchers. This study has been notified and approved by the local ethical committee.

\section{REFERENCES}

1. Kendler DL, Marin F, Zerbini CAF, et al. Effects of teriparatide and risedronate on new fractures in post-menopausal women with severe osteoporosis (VERO): a multicentre, double-blind, double-dummy, randomised controlled trial. Lancet. 2018; 391: 230-40.

2. Bone HG, Wagman RB, Brandi ML, et al. 10 years of denosumab treatment in postmenopausal women with osteoporosis: results from the phase 3 randomised FREEDOM trial and open-label extension. Lancet Diabetes Endocrinol. 2017; 5: 513-23.

3. Bouxsein ML, Eastell R, Lui L-Y, et al. Change in Bone Density and Reduction in Fracture Risk: A Meta-Regression of Published Trials. J Bone Miner Res. 2019; 34: 632-2.

4. Pedersen AB, Heide-Jørgensen U, Sørensen HT, et al. Comparison of Risk of Osteoporotic Fracture in Denosumab vs Alendronate Treatment Within 3 Years of Initiation. JAMA Netw Open. 2019; 2: e192416.

5. Kanis JA, Johnell O, Oden A, et al. FRAX and the assessment of fracture probability in men and women from the UK. Osteoporos Int. 2008; 19: 385-97.

6. Agenzia Italiana del Farmaco (AIFA). Guideline for the classification and conduction of the observational studies on medicines. Available from: https://www.agenziafarmaco.gov. it/ricclin/sites/default/files/files_wysiwyg/ files/CIRCULARS/Circular\%2031st\%20 May\%202010.pdf

7. Rossini M, Adami S, Bertoldo F, et al. Guidelines for the diagnosis, prevention and management of osteoporosis. Reumatismo. 2016; 68: 1-39.

8. Compston J, Cooper A, Cooper C, et al. UK clinical guideline for the prevention and treatment of osteoporosis. Arch Osteoporos. 2017; 12: 43.

9. Austin M, Yang Y-C, Vittinghoff E, et al. Relationship between bone mineral density changes with denosumab treatment and risk reduction for vertebral and nonvertebral fractures. J Bone Miner. Res. 2012; 27: 687-93.

10. Brown JP, Prince RL, Deal C, et al. Comparison of the effect of denosumab and alendronate on BMD and biochemical markers of bone turnover in postmenopausal women with low bone mass: a randomized, blinded, phase 3 trial. J Bone Miner Res. 2009; 24: 153-61.

11. Agenzia Italiana del Farmaco (AIFA). Nota 79 - Prescription of osteoporosis medications at risk of fragility fracture or subsequent fracture. Available from: http://www.gazzettaufficiale.it/eli/id/2015/05/20/15A03762/sg 